\title{
Some ball quotients with a Calabi-Yau model
}

Eberhard Freitag

Mathematisches Institut

Im Neuenheimer Feld 288

D69120 Heidelberg

freitag@mathi.uni-heidelberg.de
Riccardo Salvati Manni

Dipartimento di Matematica, Piazzale Aldo Moro, 2

I-00185 Roma, Italy.

salvati@mat . uniroma1.it

2012

\section{Introduction}

In a sequence of papers, cf. [FS1], [FS2], [FS3] and [CFS], we studied Siegel threefolds which admit a Calabi-Yau model. We got a long list of projective Calabi-Yau manifolds. Some of them have been new. In this paper we start to look to other kinds of modular examples. A natural class are the ball quotients which belong to the unitary group $\mathrm{U}(1,3)$. Its arithmetic subgroups are called Picard modular groups and the corresponding varieties are called Picard modular varieties. In [FS4] we determined explicitly a Picard modular variety of general type. On the regular locus of this variety there are many holomorphic three forms with known zero divisors which have been constructed as Borcherds products. Resolutions of quotients of this variety, such that the zero divisors are in the branch locus, are candidates for Calabi-Yau manifolds. The aim of this note is to treat one distinguished example for this. In fact we shall recover a known variety ([Me], Chap. 5, Sect. 6) given by the equations

$$
X_{0} X_{1} X_{2}=X_{3} X_{4} X_{5}, \quad X_{0}^{3}+X_{1}^{3}+X_{2}^{3}=X_{3}^{3}+X_{4}^{3}+X_{5}^{3}
$$

as a Picard modular variety with respect to a certain Picard modular group $G^{\prime}$. As it has been explained in [Me], this variety has a projective small resolution which is a rigid Calabi-Yau manifold $\left(h^{12}=0\right)$ with Euler number 72. Moreover a certain group acts on this variety, fixing the holomorphic differential 3 -form. We will show that this group is modular in the sense that it is given by a subgroup of the normalizer of $G^{\prime}$ in the full modular group. 


\section{Differential forms}

Let $V$ be a finite dimensional complex vector space which is equipped with a hermitean form $\langle\cdot, \cdot\rangle$ of signature $(1, n)$. A line (=one dimensional sub-vectorspace of $V$ ) is called positive if it is represented by an element of positive norm $(\langle z, z\rangle>0)$. We denote by $\mathcal{B}=\mathcal{B}(V) \subset P(V)$ the set of all positive lines. (As usual $P(V)$ denotes the projective space of $V$, i.e. the set of all lines in $V$.) The unitary group $\mathrm{U}(V) \cong \mathrm{U}(1, n)$ acts on $\mathcal{B}$ as group of biholomorphic automorphisms.

We choose a sub-vector space $W \in V$ of codimension one which contains no vector of positive norm. We also consider a vector $e \in V$ such that

$$
V=\mathbb{C} e \oplus W
$$

If $v=C e+w, w \in W$, is a vector of positive norm, then $C \neq 0$. Hence we can normalize the line $\mathbb{C} v$ such that $C=1$. This gives an embedding of $\mathcal{B}$ into $W$. The linear action of $\mathrm{U}(V)$ on $\mathcal{B}(V)$ gives a rational action $z \mapsto g\langle z\rangle$ on the image of $\mathcal{B}$ in $W$. If we write $g(e+z)=C e+w$, then $g\langle z\rangle=w / C$. We have

$$
g(e+z)=j(g, z)(e+g\langle z\rangle) \quad \text { where } \quad j(g, z):=C .
$$

The function $j(g, z)$ is an automorphy factor. We call it the canonical automorphy factor. Another automorphy factor is the Jacobian.

2.1 Lemma. The Jacobian of the transformation $z \mapsto g\langle z\rangle$ for $g \in \mathrm{U}(V)$ is

$$
\operatorname{det}(g) j(g, z)^{-(n+1)}
$$

Proof. The proof can be given for reflections by a somewhat tedious computation.

Let $M$ be a lattice in $V$, i.e. a discrete subgroup with compact quotient. We assume that $M$ admits complex multiplication. Then the set of all complex numbers $a \in \mathbb{C}$ such that $a M \subset M$ is an order in an imaginary number field. The Picard modular group $\mathrm{U}(M)$ is the subgroup of $\mathrm{U}(V)$ that preserves $M$.

We recall the notion of a modular form of integral weight $k$. For this we consider the inverse image $\tilde{\mathcal{B}}$ of $\mathcal{B}$ in $V-\{0\}$. It consists of all $z \in V$ with $\langle z, z\rangle>0$. This is a connected open subset.

Let $G \subset \mathrm{U}(M)$ be a subgroup of finite index and let $v: G \rightarrow \mathbb{C}^{\cdot}$ be a character. 
A modular form of weight $k$ on $G$ with respect to a $v$ is a holomorphic function $f: \tilde{\mathcal{B}} \rightarrow \mathbb{C}$ with the following properties:

1) $f(t z)=t^{-k} f(z)$ for all $t \in \mathbb{C}^{\circ}$,

2) $f(\gamma z)=v(\gamma) f(z)$ for all $\gamma \in G$,

3) $f$ is regular at the cusps.

We denote this space of modular forms by $[G, k, v]$ or simply by $[G, k]$ when $v$ is trivial. The ring of modular forms

$$
A(G)=\bigoplus[G, k]
$$

is a finitely generated algebra. From the theory of Baily and Borel follows that $A(G)$ is a finitely generated algebra whose associated projective variety can be identified with the compactification $X(G)$ of $\mathcal{B} / G$ by (finitely many) cusps,

$$
\operatorname{proj} A(G) \cong X(G) .
$$

From Lemma 2.1 we obtain the following result.

2.2 Lemma. There is a one-to-one correspondence between $G$-invariant InvDif differential forms of top degree $n$ on $\mathcal{B}$ and modular forms of weight $n+1$ and multiplier system $v(\gamma)=\operatorname{det}(\gamma)^{-1}$.

We mention a well-known general fact. A $G$-invariant differential form of top degree $n$ extends holomorphically to a smooth compactification of $\mathcal{B} / G$ if and only if the associated modular form is cuspidal (compare [Fr], Satz III.2.6).

\section{A special group}

We equip $V=\mathbb{C}^{4}$ with the hermitian form

$$
\langle a, b\rangle=\bar{a}_{1} b_{2}+\bar{a}_{2} b_{1}-\bar{a}_{3} b_{3}-\bar{a}_{4} b_{4} .
$$

Let $\mathcal{E}$ be the ring of Eisenstein numbers. We consider the lattice $M=\mathcal{E}^{4}$. We use the notation

$$
G_{3}=\mathrm{U}(M) .
$$

We also consider the principal congruence subgroups

$$
G_{3}[a]=\operatorname{kernel}\left(G_{3} \longrightarrow \mathrm{GL}(4, \mathcal{E} / a)\right) \quad(a \in \mathcal{E}, a \neq 0) .
$$

In the paper [FSM] the ring of modular forms of $G_{3}[3]$ has been determined. We recall some of the main results. There are 15 forrms $B_{1}, \ldots, B_{15}$ of weight 1 and 10 cusp forms $C_{1}, \ldots, C_{10}$ of weight 10 . These 25 forms generate the ring 
of modular forms $A\left(G_{3}[3]\right)$. The relations between the generators have been determined:

$$
\operatorname{dim}\left[G_{3}[3], k\right]= \begin{cases}0 & \text { for } k<0 \\ 1 & \text { for } k=0 \\ 15 & \text { for } k=1 \\ 130 & \text { for } k=2 \\ 750 & \text { for } k=3 \\ 3115 & \text { for } k=4 \\ -1377+(8019 / 2) k-2187 t k^{2}+(729 / 2) k^{3} & \text { for } k>4\end{cases}
$$

and

$$
\operatorname{dim}\left[G_{3}[3], k\right]-\operatorname{dim}\left[G_{3}[3], k\right]_{0}= \begin{cases}15 & \text { for } k=1 \\ 120 & \text { for } k=2 \\ 405 & \text { for } k=3 \\ 765 & \text { for } k=4 \\ 810 & \text { for } k>4\end{cases}
$$

Basic elements of $G_{3}$ are reflections along vectors $b$ of norm $\langle b, b\rangle=-1$. They are defined by

$$
a \longmapsto a-(1-\eta) \frac{\langle b, a\rangle}{\langle b, b\rangle} b
$$

where $\eta$ is a 6 th root of unity (hence a power of $-\zeta$ ). They transform $b$ to $\eta b$ and act as identity on the orthogonal complement of $b$. Their order equals the order of $\eta$. We call them biflections, triflections or hexflections corresponding to the order of $\eta$.

By a short mirror in $\tilde{\mathcal{B}}$ we understand the fixed point set of a triflection. The short mirrors decompose under $G_{3}[\sqrt{-3}]$ into 15 orbits.

$\begin{array}{lllll}1 & 2 & 3 & 4 & 5 \\ (0,0,1,0) & (0,0,0,1) & (1,0,1,0) & (1,0,-1,0) & (1,0,0,1) \\ 6 & 7 & 8 & 9 & 10 \\ (1,0,0,-1) & (0,1,1,0) & (0,1,-1,0) & (0,1,0,1) & (0,1,0,-1) \\ 11 & 12 & 13 & 14 & 15 \\ (\zeta,-1,1,1) & (\zeta,-1,1,-1) & (\zeta,-1,-1,1) & (\zeta,-1,-1,-1) & (\zeta, 1,0,0)\end{array}$

We recall the basic properties of the forms $B_{1}, \ldots, B_{15}$. Their zero divisor (considered $\bmod G_{3}[3]$ ) consist of three short mirrors which are described in the following list:

$(1,2,15),(2,4,8),(2,3,7),(1,6,10),(1,5,9),(12,13,15), \quad(11,14,15),(4,6,11)$, $(4,5,12),(8,10,14),(8,9,13),(3,6,13),(3,5,14),(7,10,12),(7,9,11)$. 
We also recall the definition of the cusp forms $C_{i}$.

$$
\begin{aligned}
C_{1} & =B_{2} B_{4} B_{15} / B_{8}, \\
C_{2} & =B_{2} B_{13} B_{15} / B_{3}, \\
C_{3} & =B_{3} B_{6} B_{10} / B_{14}, \\
C_{4} & =B_{3} B_{5} B_{8} / B_{15}, \\
C_{5} & =B_{8} B_{13} B_{14} / B_{9}, \\
C_{6} & =B_{5} B_{7} B_{14} / B_{15}, \\
C_{7} & =B_{2} B_{6} B_{15} / B_{11}, \\
C_{8} & =B_{1} B_{8} B_{11} / B_{2}, \\
C_{9} & =B_{6} B_{13} B_{15} / B_{7}, \\
C_{10} & =B_{2} B_{4} B_{6} / B_{1} .
\end{aligned}
$$

Each of them vanishes along 6 short mirrors (counted mod $G_{3}[3]$ ). For example $C_{1}^{2}$ vanishes along the mirrors with the digits $1,2,7,8,9,10$. We denote by $G^{\prime}$ the group which is generated by $G_{3}[3]$ and the 6 triflections corresponding to the above 6 short mirrors.

From 2.2 follows that $C_{1}^{2}$ defines a $G^{\prime}$-invariant differential form. Moreover its zeros (of order two) disappear in the quotient $\mathcal{B} / G^{\prime}$ due to the ramification. Hence there is a chance that a suitable desingularization gives a Calabi-Yau manifold. We will show now that this is actually the case.

3.1 Lemma. The index of the group $G_{3}[3]$ in $G^{\prime}$ is 486. It contains the IndGr negative of the identity, The covering degree of $X\left(G_{3}[3]\right) \rightarrow X\left(G^{\prime}\right)$ is 243 .

Proof. The proof can be given by means of a direct computation with the help of a computer.

The forms $B_{6}, B_{7}, B_{8}, B_{9}, B_{12}, B_{13}$ do not vanish along one of the six short mirrors. Hence they have trivial multiplier with respect to $G^{\prime}$.

3.2 Theorem. The six modular forms above generate the ring $A\left(G^{\prime}\right)$. Defin- Lems ing relations are

$$
B_{7} B_{9} B_{12}=B_{6} B_{8} B_{13}, \quad B_{6}^{3}+B_{7}^{3}-B_{8}^{3}+B_{9}^{3}-B_{12}^{3}-B_{13}^{3}=0 .
$$

The associated variety $\mathcal{X}$ has 108 singularities which are nodes.

Proof. From the description of the zeros one can derive that the 6 forms have no joint zero. By a result of Hilbert, the ring $A\left(G^{\prime}\right)$ is integral over the subring $\mathbb{C}\left[B_{6}, B_{7}, B_{8}, B_{9}, B_{12}, B_{13}\right]$. The relations described in the theorem generate a prime ideal. A dimension argument shows that they are defining relations between the 6 forms. The Hilbert polynomial of the subring can be computed. The result is $\left(27 t^{2}+9 t^{3}\right) / 3$ !. Its highest coefficient is $3 / 2$. We 
also know that the highest coefficient of the Hilbert polynomial of $A\left(G^{\prime}\right)$ is $729 / 2$. The quotient of the two highest coefficients, 243, equals the covering degree $\left[G^{\prime}: G_{3}[3]\right]$ of $X\left(G^{\prime}\right) \rightarrow X\left(G_{3}[3]\right)$. So we see that $A\left(G^{\prime}\right)$ and the subring $\mathbb{C}\left[B_{6}, B_{7}, B_{8}, B_{9}, B_{12}, B_{13}\right]$ have the same field of fractions. So $A\left(G^{\prime}\right)$ is the normalization of this subring. One can check that the relations in the theorem define a normal ring. In fact it is a complete intersection and regular in codimension 2. This finishes the proof.

We introduce variables $X_{0}, \ldots, X_{5}$ and consider the homomorphism

$$
\mathbb{C}\left[X_{0}, \ldots, X_{5}\right] \longrightarrow A\left(G^{\prime}\right)
$$

which is defined by

$X_{0} \longmapsto-B_{6}, X_{1} \longmapsto B_{8}, X_{2} \longmapsto B_{13}, X_{3} \longmapsto B_{7}, X_{4} \longmapsto B_{9}, X_{5} \longmapsto-B_{12}$.

Then the defining relations get

$$
X_{0} X_{1} X_{2}=X_{3} X_{4} X_{5}, \quad X_{0}^{3}+X_{1}^{3}+X_{2}^{3}=X_{3}^{3}+X_{4}^{3}+X_{5}^{3}
$$

This is a very well-known variety. We learned from [Me], Chap. 5, Sect. 6, that it has 108 singularities which are all nodes. They correspond to certain cusps in the ball-model. Moreover there is a projective small resolution which is a rigid Calabi-Yau manifold $\left(h^{12}=0\right)$ with Euler number 72. The Picard number is $h^{11}=36$.

3.3 Theorem. The variety $\mathcal{X}$ defined in $P^{5} \mathbb{C}$ as intersection of two cubics

$$
X_{0} X_{1} X_{2}=X_{3} X_{4} X_{5}, \quad X_{0}^{3}+X_{1}^{3}+X_{2}^{3}=X_{3}^{3}+X_{4}^{3}+X_{5}^{3}
$$

can be identified with (compactified) ball quotient $X\left(G^{\prime}\right)$ with respect to the group $G^{\prime}$. This gives an example of a three dimensional ball-quotient which admits a Calabi-Yau model.

There is an obvious group $H$ of automorphisms of order 5832 acting on this variety. First we can consider permutations of the variables $X_{0}, X_{1}, X_{2}$ and $X_{3}, X_{4}, X_{5}$ separately and by interchanging these two blocs. This gives a group of order 72. Then we consider transformations $X_{\nu} \mapsto \zeta_{\nu} X_{\nu}$ where $\zeta_{\nu}$ are third roots of unity with the property

$$
\zeta_{0} \zeta_{1} \zeta_{2}=\zeta_{3} \zeta_{4} \zeta_{4}
$$

Taking into account that $(\zeta, \ldots, \zeta)$ acts as identity, this gives us group of order 81 acting on the projective variety. Both types generate a group of order $72 \cdot 81=5832$ as has been stated. In fact, these automorphisms are modular. 
3.4 Remark. Let $N^{\prime}$ be the normalizer of $G^{\prime}$ in the full modular group. NormStrich There is a natural surjective homomorphism

$$
N^{\prime} \longrightarrow H
$$

which is compatible with the actions of $N^{\prime}$ on $X\left(G^{\prime}\right)$ and of $H$ on $\mathcal{X}$. The kernel of this homomorphism is generated by $G^{\prime}$ and by the transformation "multiplication by $\zeta$ ".

Proof. Since we know the action of the full modular group on the generators, the statement can by checked by means of a direct computation. We did it with the help of a computer.

We want to determine the subgroup that leaves the Calabi-Yau form invariant. The Calabi-Yau form (given in the modular picture by the modular form $C_{1}^{2}$ ) can be described in the model $\mathcal{X}$ as follows. Consider the differential form

$$
\omega=\frac{\sum_{i=0}^{5} z_{i} d z_{0} \wedge \ldots \wedge \widehat{d z}_{i} \wedge \ldots \wedge d z_{5}}{\left(z_{0} z_{1} z_{2}-z_{3} z_{4} z_{5}\right)\left(z_{0}^{3}+z_{1}^{3}+z_{2}^{3}-z_{3}^{3}-z_{4}^{3}-z_{5}^{3}\right)}
$$

This is a meromorphic differential form on $P^{5} \mathbb{C}$ with poles on the union of the two cubics. Taking the Poincaré residue we get a the Calabi-Yau form on $\mathcal{X}$. The group $H$ extends to $P^{5} \mathbb{C}$ in an obvious way. It acts on $\omega$ with a character $\chi$. This character can be computed easily:

3.5 Remark. The character $\chi$ of $H$ that describes the action of $H$ on the AutH Calabi-Yau form is defined by the following properties:

1) For a permutation of $X_{0}, X_{1}, X_{2}$ and similarly of $X_{3}, X_{4}, X_{5}$ it is the sign of the permutation.

2) For the permutation $X_{0} \leftrightarrow X_{3}, X_{1} \leftrightarrow X_{4}, X_{2} \leftrightarrow X_{5}$ it is -1 .

3) For a transformation $X_{\nu} \mapsto \zeta_{\nu} X_{\nu}$ where $\zeta_{\nu}$ are third roots of unity with the property $\zeta_{0} \zeta_{1} \zeta_{2}=\zeta_{3} \zeta_{4} \zeta_{5}$ the value of $\chi$ is $\zeta_{0} \zeta_{1} \zeta_{2}$. The kernel of $\chi$ is a subgroup of index 6 in $H$ which has the order 972.

It might be worthwhile to study subgroups of the kernel of $\chi$ such that the corresponding quotient of $\mathcal{X}$ admits a Calabi-Yau model.

\section{References}

[CFS] Cynk, S., Freitag, E., Salvati Manni, R.: The geometry and arithmetic of a Calabi-Yau Siegel threefold, Int. Jour. Math. 29, 1561-1583 (2011) (arXiv: 1004.2997) 
[Fr] Freitag, E.: Siegelsche Modulfunktionen, Grundlehren der mathematischen Wissenschaften, Bd. 254. Berlin-Heidelberg-New York: Springer (1983)

[FS1] Freitag, E., Salvati Manni, R.: Some Siegel threefolds with Calabi-Yau model, Ann. Scuola Norm. Sup. Pisa Cl. Sci. (5). Vol IX, 833-850 (2010) (arXiv: 0905.4150)

[FS2] Freitag, E., Salvati Manni, R.: Some Siegel threefolds with Calabi-Yau model II, To appear on Kyungpook Math. Journal] (arXiv: 1001.0324)

[FS3] Freitag, E., Salvati Manni, R.: On Siegel three folds with a projective CalabiYau model, Communications in Number Theory and Physics. Volume 5, Number 3 p.713-750 ( 2011) (arXiv: 1103.2040)

[FS4] Freitag, E., Salvati Manni, R.: A three dimensional ball quotient, preprint (2012)(arXiv: 1201.0131)

[Me] Meyer, C.: A dictionary of modular threefolds, thesis, University of Mainz, Fachbereich Mathematik und Informatik (2005) 